\title{
THERMAL ENERGY EXTRACTION FROM A GEOTHERMAL RESERVOIR: NUMERICAL AND ANALYTICAL MODELING ANALYSIS
}

Jeremy Patterson, Michael Cardiff, Herb Wang, Kurt Feigl, and PoroTomo Team 


\section{ACKNOWLEDGEMENTS}

\section{Collaborators (PoroTomo Team):}

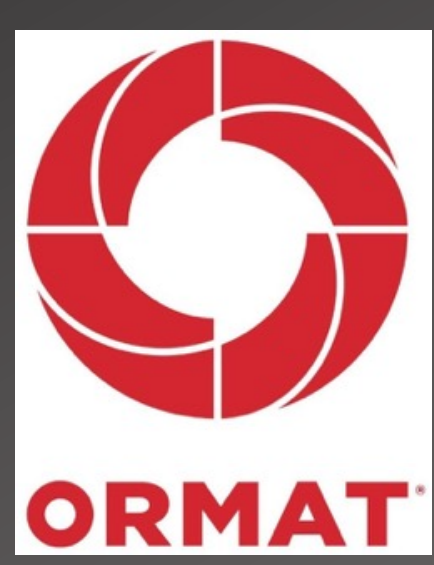

\section{Support:}

The work presented herein was funded in part by the Office of Energy Efficiency and Renewable Energy (EERE), U.S. Department of Energy, under Award Numbers DE-EE0006760 and DE-EE0005510.

ORMAT Technologies, Inc. generously provided field site access, safety training, and data sharing to support this work.

\section{Additional Support:}

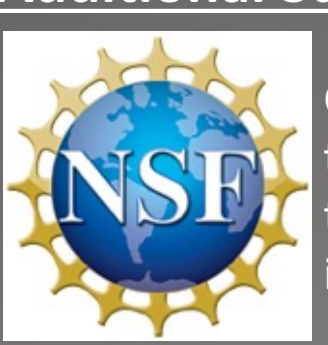

Cardiff: EAR-1654649 “CAREER: Understanding

transport processes in fractured sedimentary rock

through multi-frequency and multi-method

investigations"

\section{$\mathbb{1}$ University of Nevada, Reno}

COLORADO

Lawrence Berkeley EARTH ENERGY EN VIRONMENT

\section{TT TEMPLE TESYTY}




\section{OUTLINE}

- PoroTomo Overview

- Study Area

- Thermal Characterization

- Hydraulic Characterization

- Conclusions

• Future Work 
Big Picture Question:

- Can we constrain the thermal sustainability of a geothermal reservoir?

First Order Questions:

- Can we estimate the relevant thermal and hydraulic reservoir properties?

- How does fluid move through the reservoir?

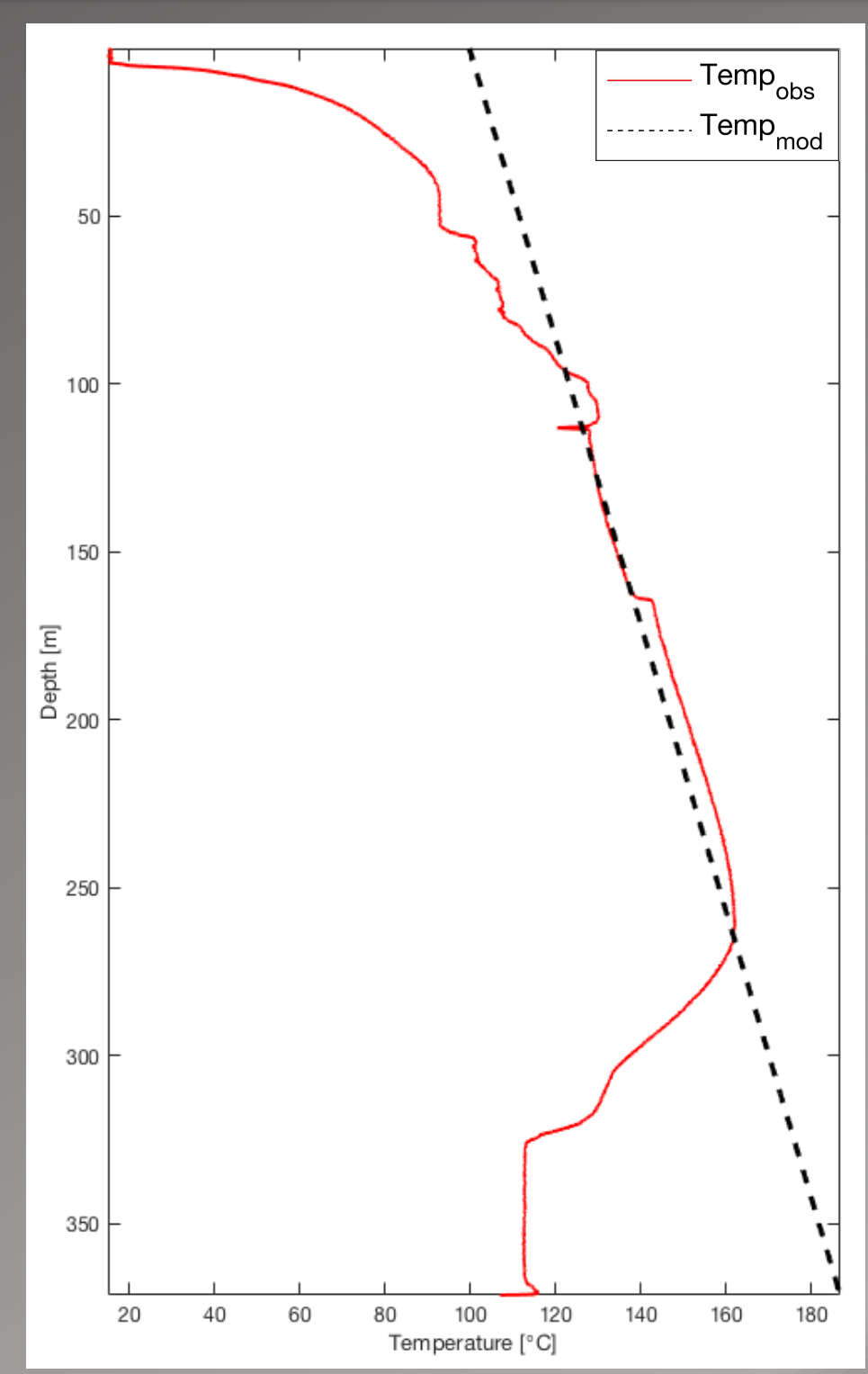


Poroelastic Tomography by Adjoint Inverse Modeling of Data from Seismology, Geodesy, and Hydrology

- Project Objective

- "...assess an integrated technology for characterizing and monitoring changes in the rock mechanical properties of an EGS reservoir in three dimensions with a spatial resolution better than 50 meters." (http://geoscience.wisc.edu/feigl/porotomo/) 


\section{STUDY AREA}

Brady Geothermal Field

- $75 \mathrm{~km}$ NE of Reno

- NE-SW trending high angle normal faults

- Shallow sedimentary units

- Deeper volcanic units

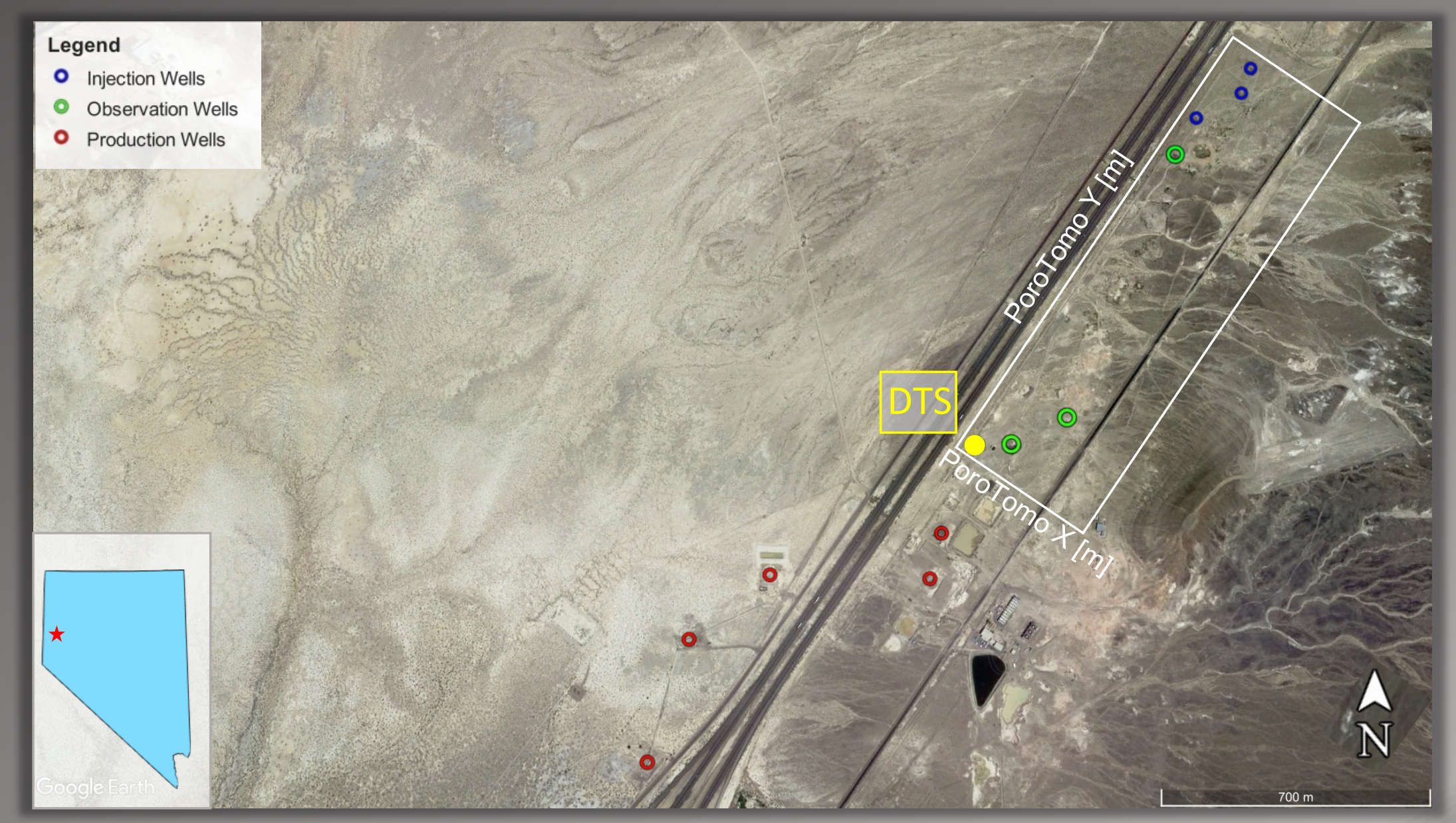




\section{THERMAL CHARACTERIZATION}

\section{Borehole DTS}

- Sampling interval

- Spatial - 0.12 [m]

- Temporal - 60 [s]

- Borehole temperature recovery

- Inverted geotherm below 260 m

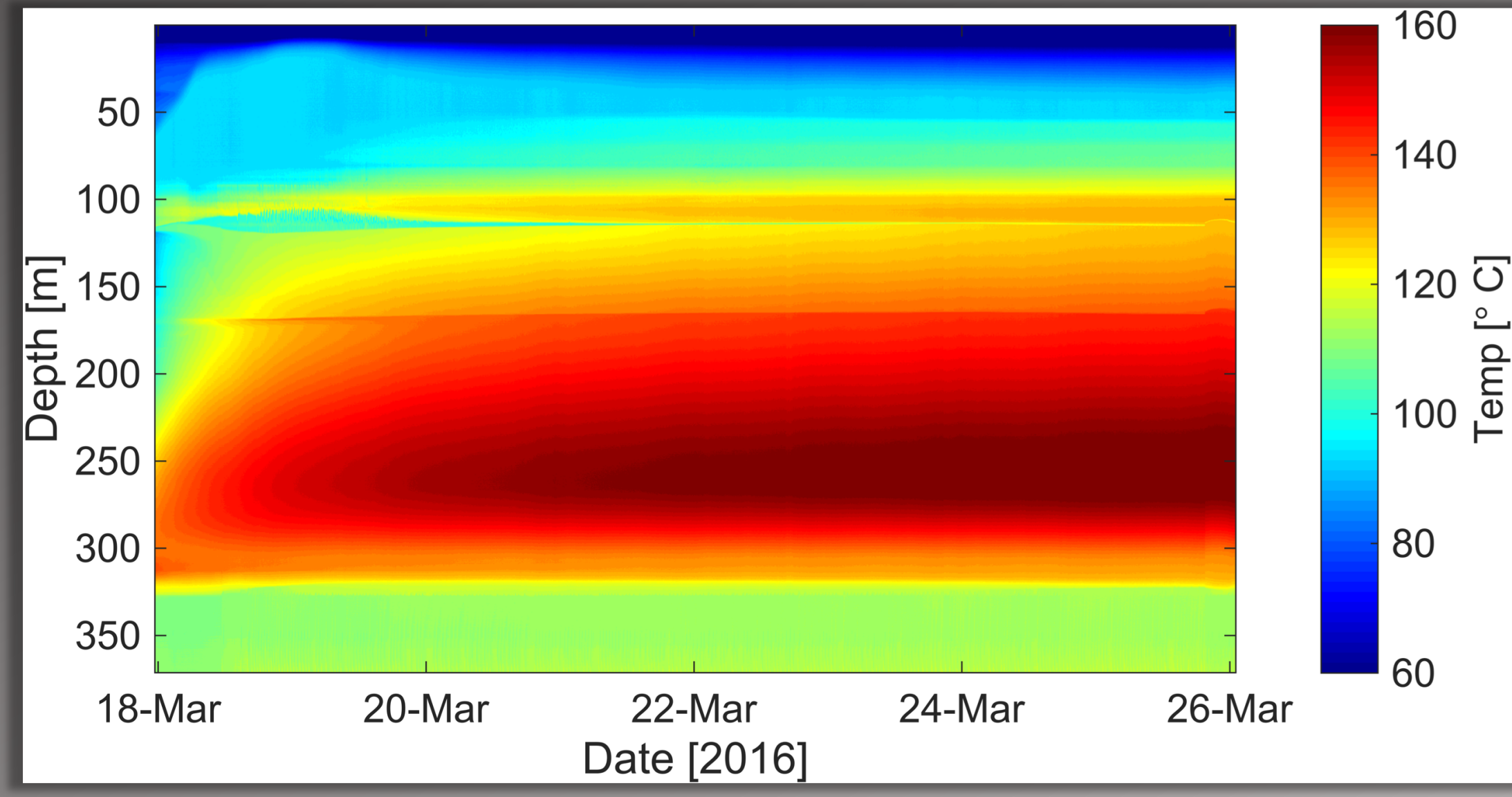




\section{THERMAL CHARACTERIZATION}

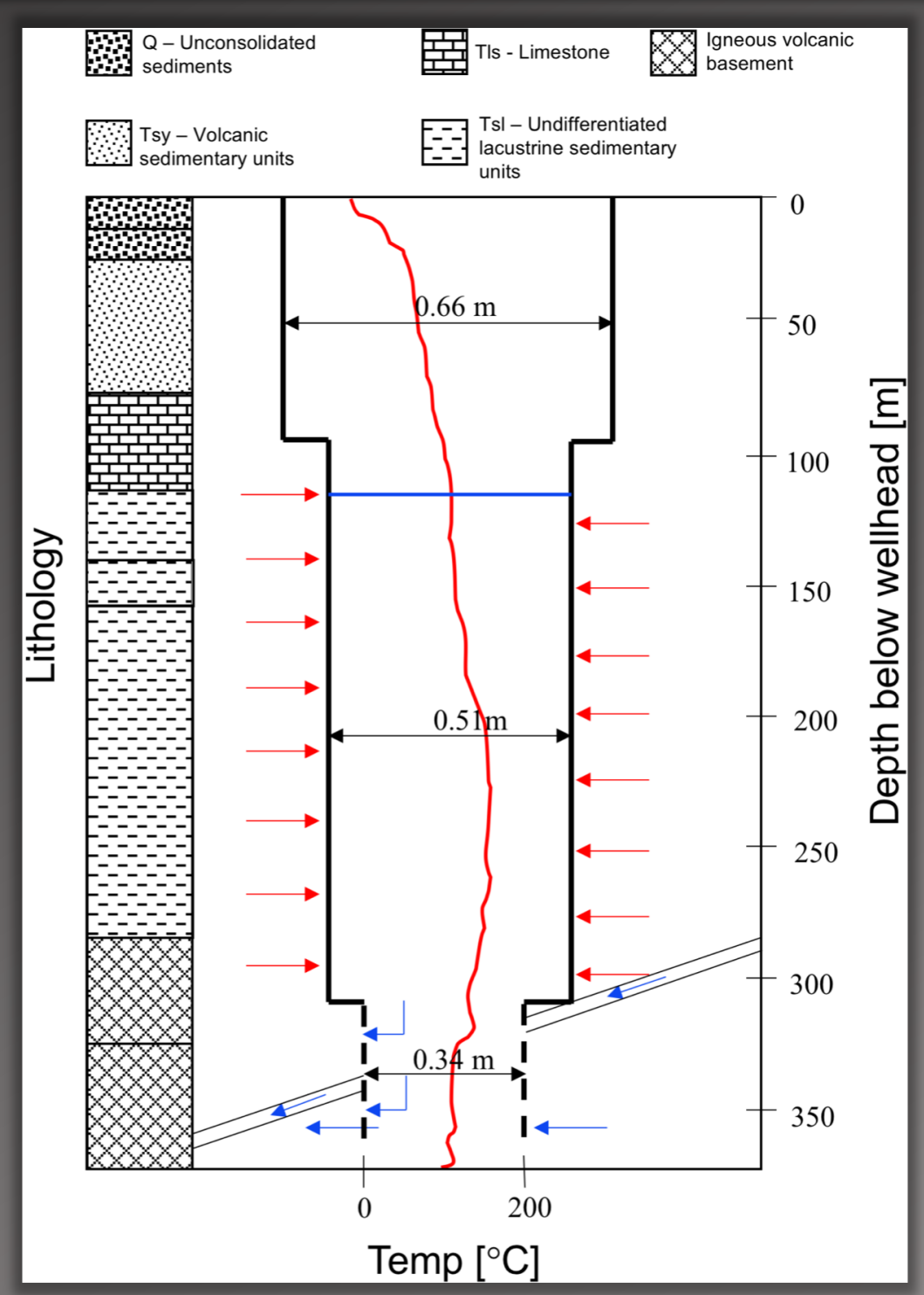

\section{Borehole schematic}

- Vertical temperature profile along borehole

- Radial heat diffusion into borehole

- Fluid filled and cased portion of borehole ( 125 - 300 m depth)

- Conceptual model for numerical heat transfer model 


\section{THERMAL CHARACTERIZATION}

\section{Parameter estimation}

\section{results:}

- Thermal diffusivity depth profile

- Major changes correlated with lithologic changes

- Estimated diffusivity lower than lab reported values

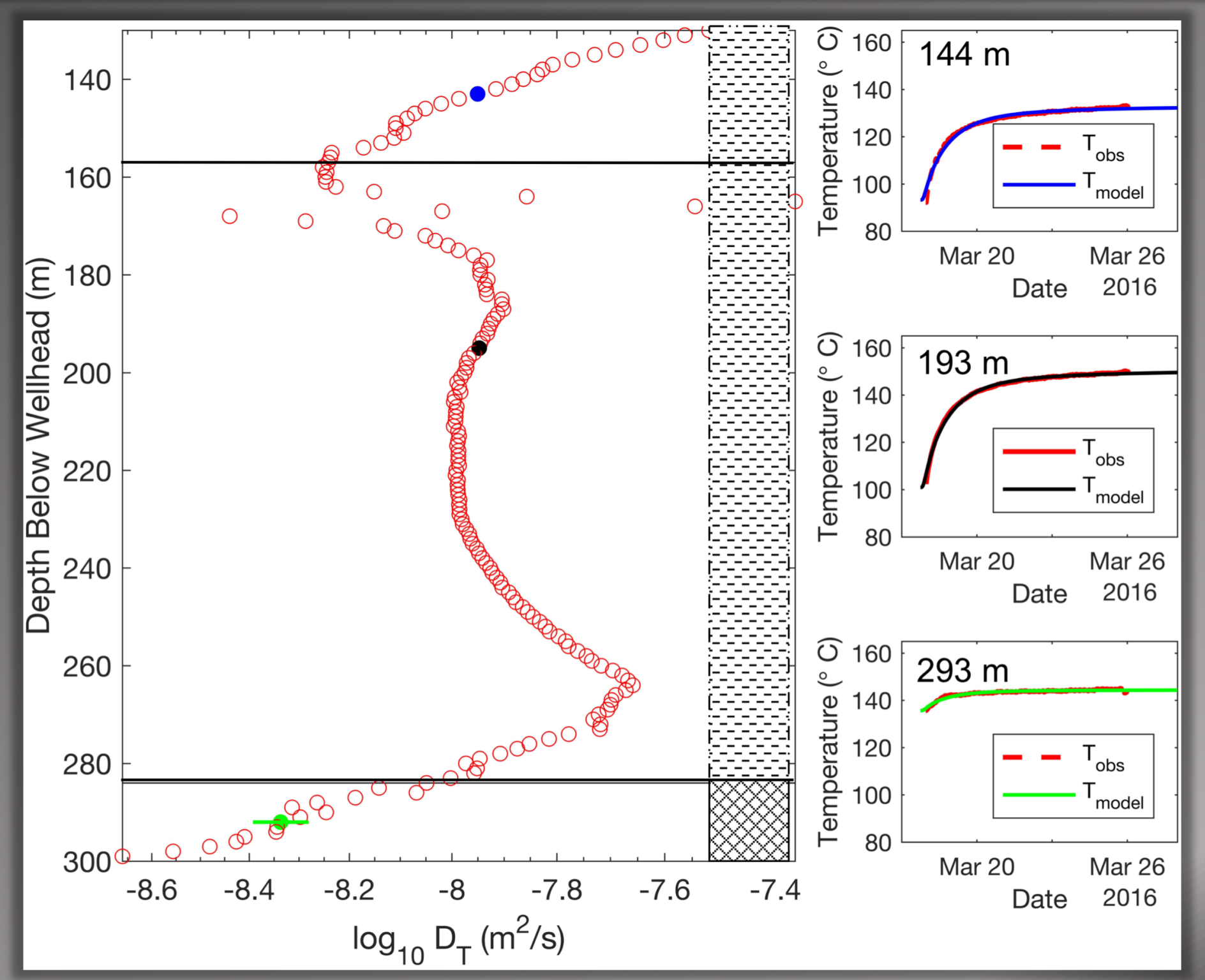




\section{SUBSURFACE FLUID FLOW}

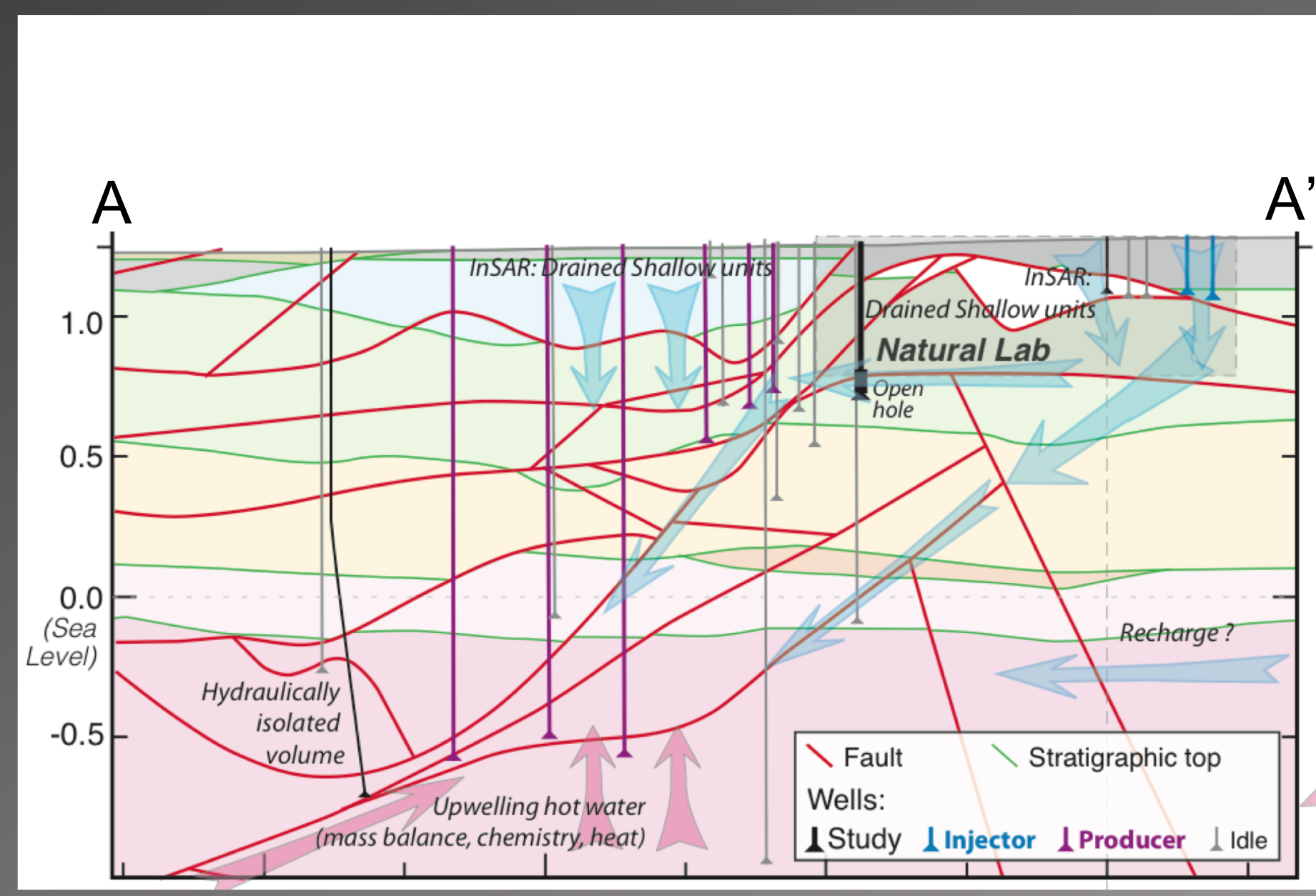




\section{SUBSURFACE FLUID FLOW}

\section{Evidence for fault-driven groundwater flow: \\ - Borehole advection}
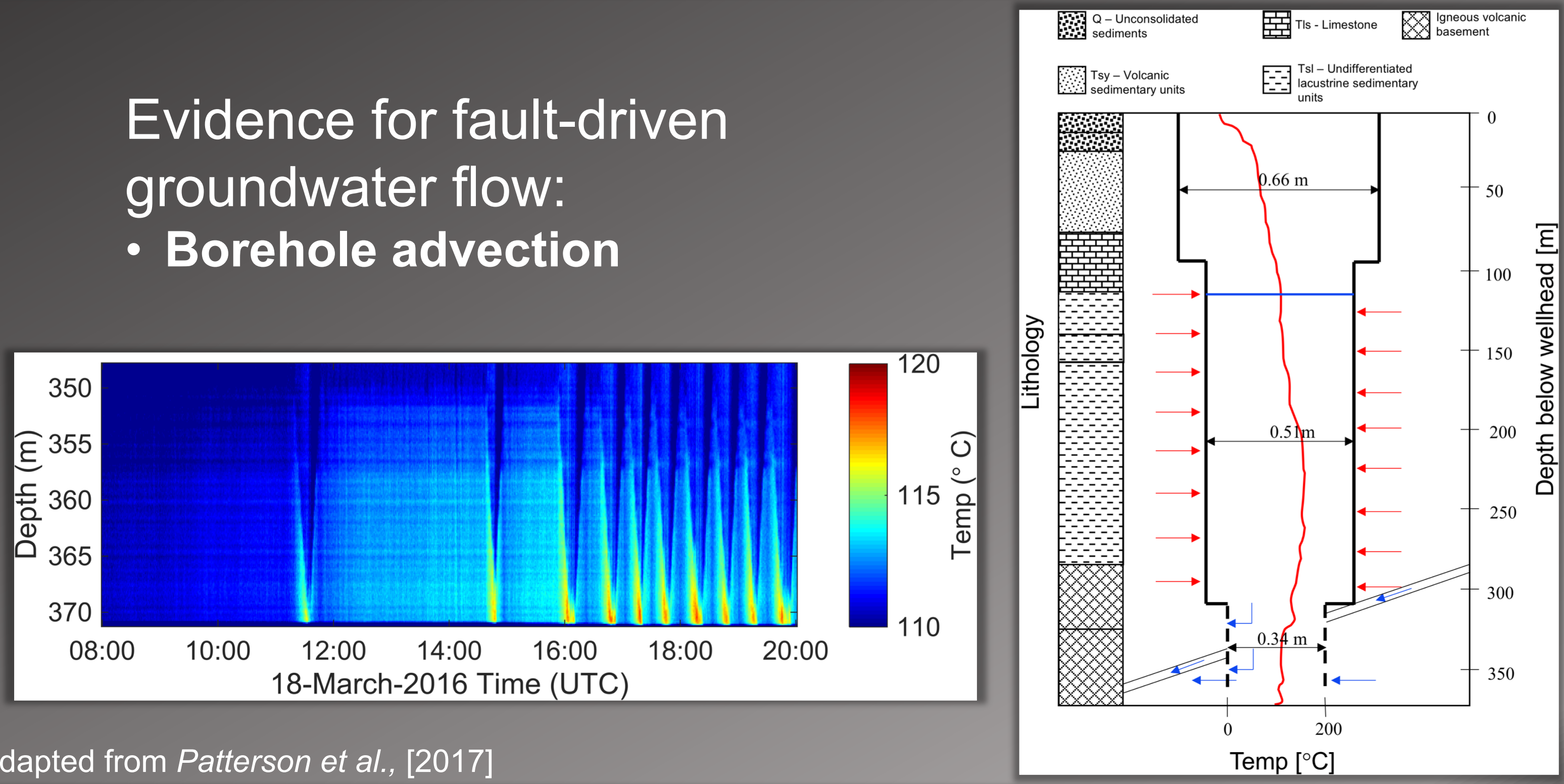


\section{SUBSURFACE FLUID FLOW}

Evidence for fault-driven groundwater flow:

- Borehole advection

- Ground subsidence

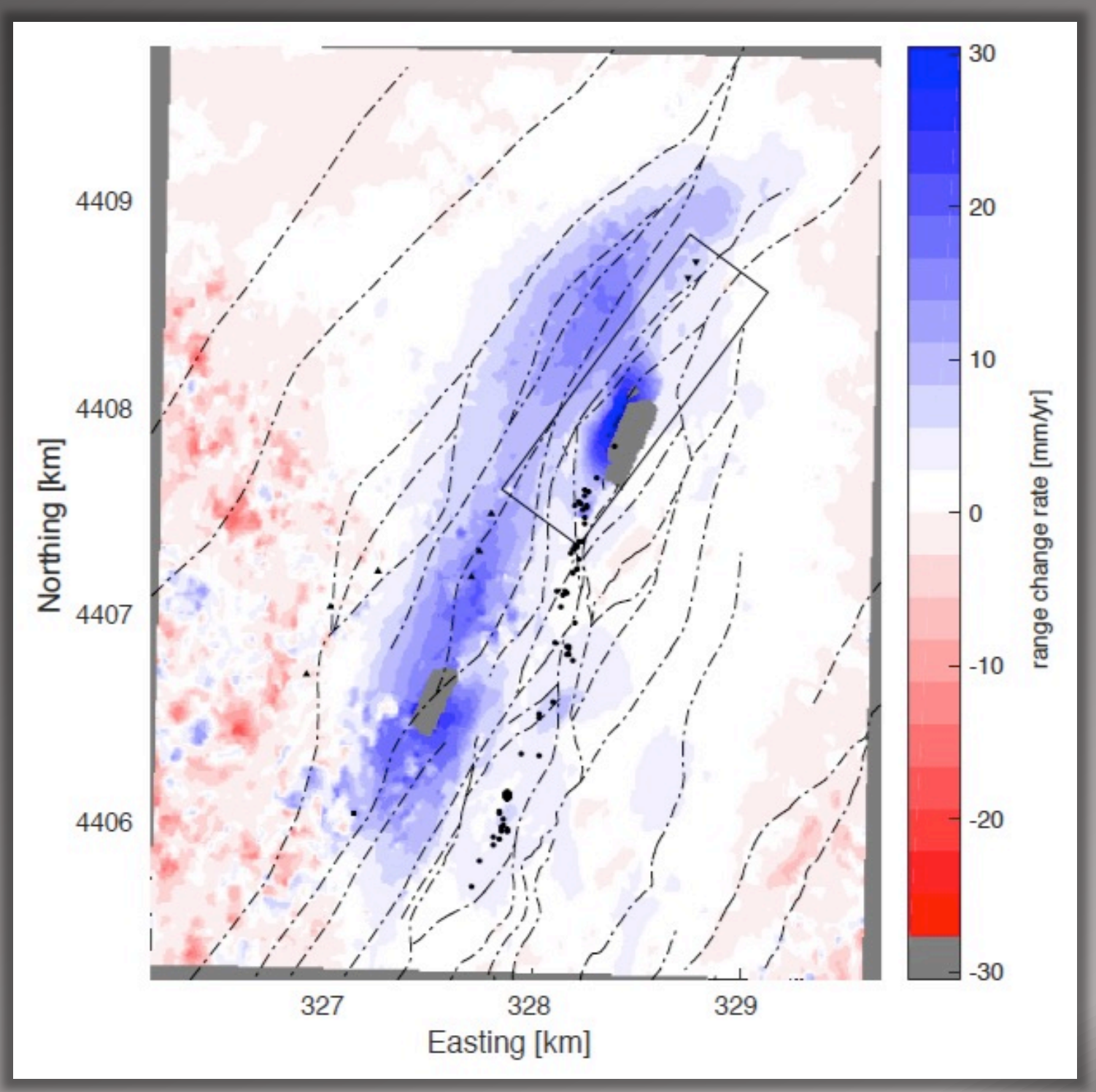




\section{SUBSURFACE FLUID FLOW}

Evidence for fault-driven groundwater flow:

- Borehole advection

- Ground subsidence

- Hydrothermal Deposits

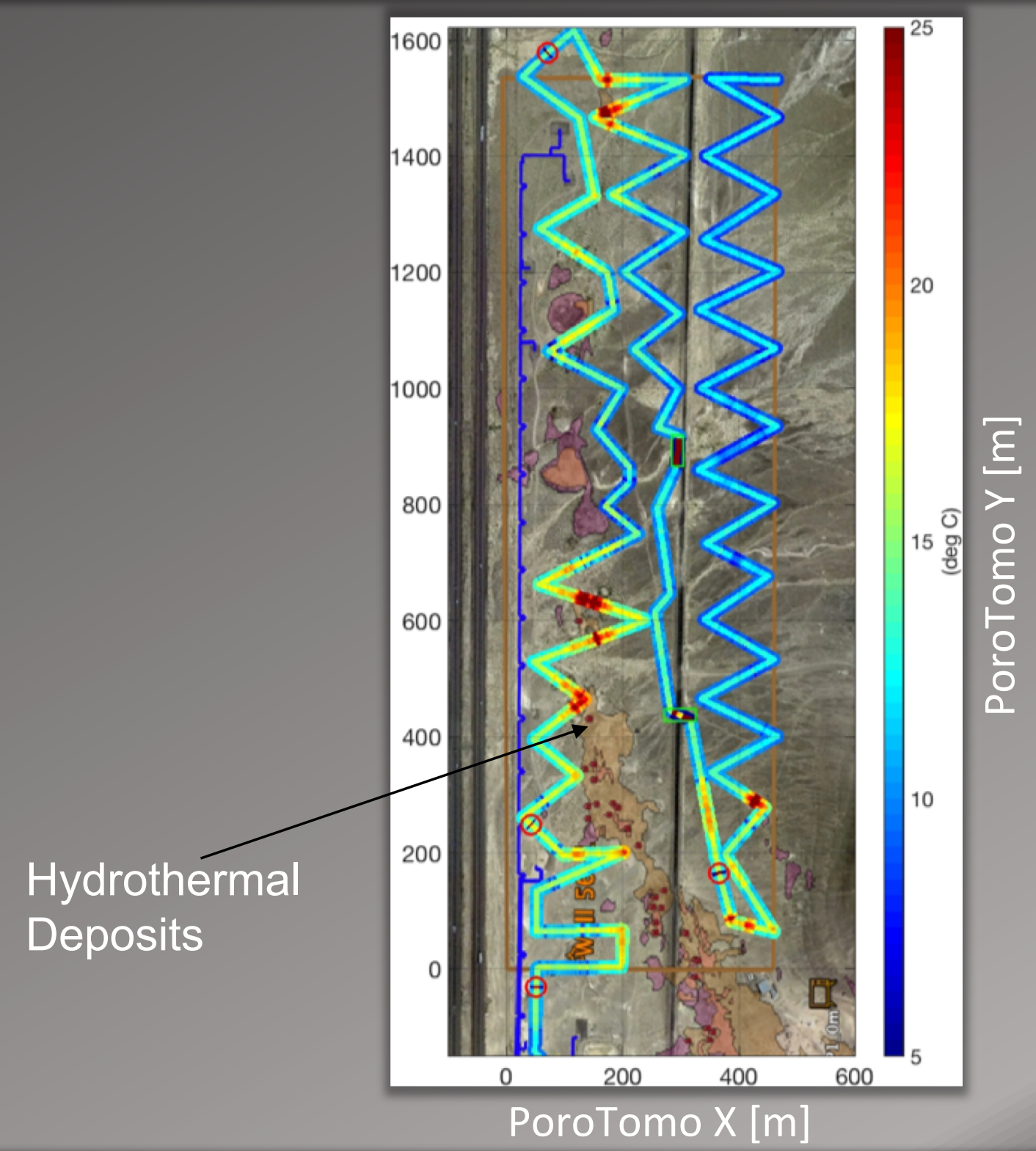




\section{HYDRAULIC CHARACTERIZATION}

Groundwater Flow Model

- 7 km x 13 km x 6 km

- Discretization

- 3 Hydraulic Property Zones

- Zoned Parameter Estimation

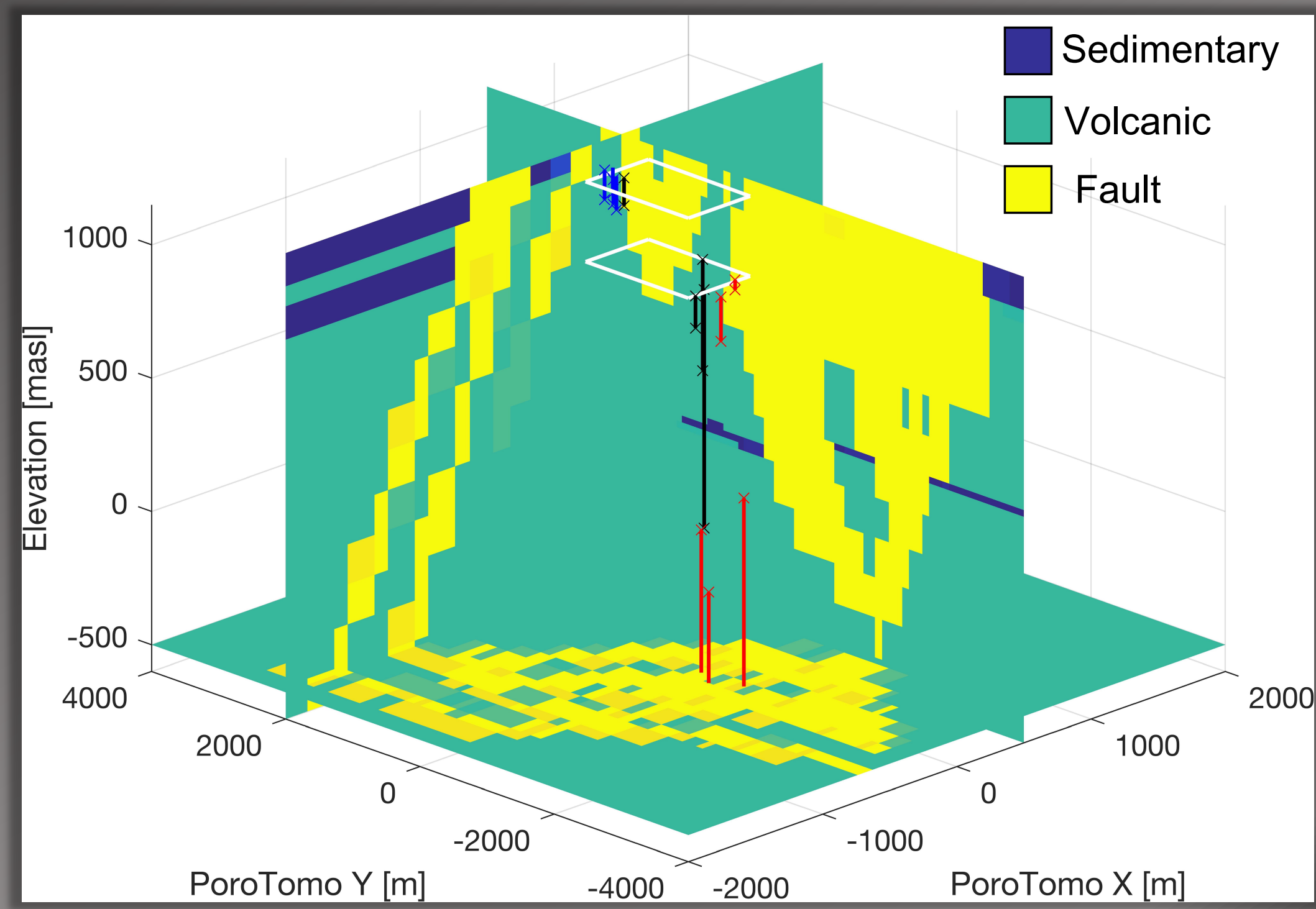




\section{HYDRAULIC CHARACTERIZATION}
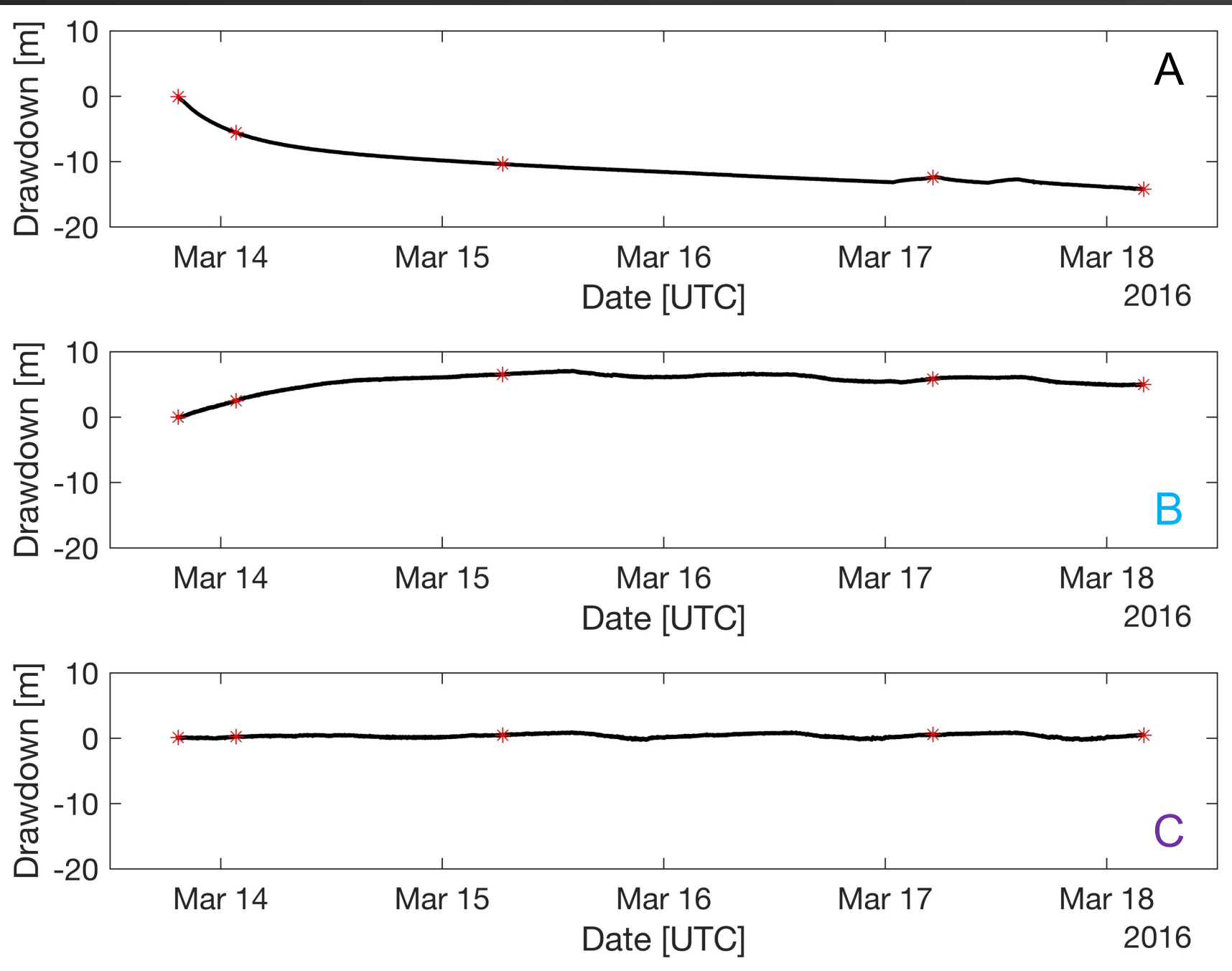


\section{HYDRAULIC CHARACTERIZATION}

\begin{tabular}{|c|c|c|}
\hline Lithology & $\mathrm{K}[\mathrm{m} / \mathrm{s}]$ & $\mathrm{S}_{\mathrm{s}}\left[\mathrm{m}^{-1}\right]$ \\
\hline Sedimentary & $4.1 \times 10^{-2}$ & $7.0 \times 10^{-8}$ \\
\hline Volcanic & $2.7 \times 10^{-4}$ & $3.5 \times 10^{-8}$ \\
\hline Fault & $1.7 \times 10^{-8}$ & $1.8 \times 10^{-6}$ \\
\hline
\end{tabular}

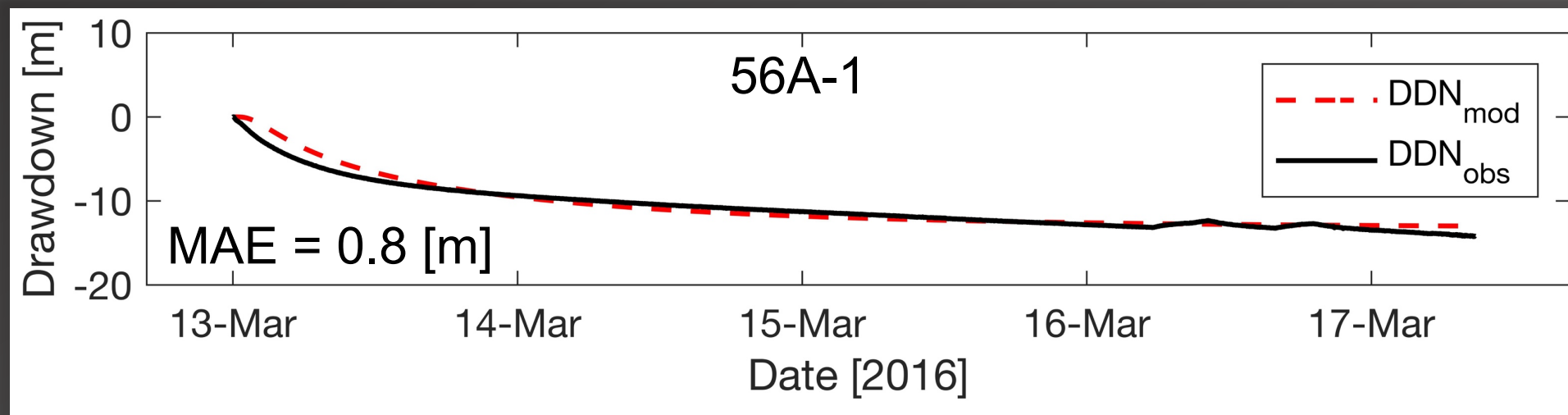

\section{Parameter estimates}

indicate:

- Specific storage estimates indicate confined behavior

- Faults may act as barrier to flow

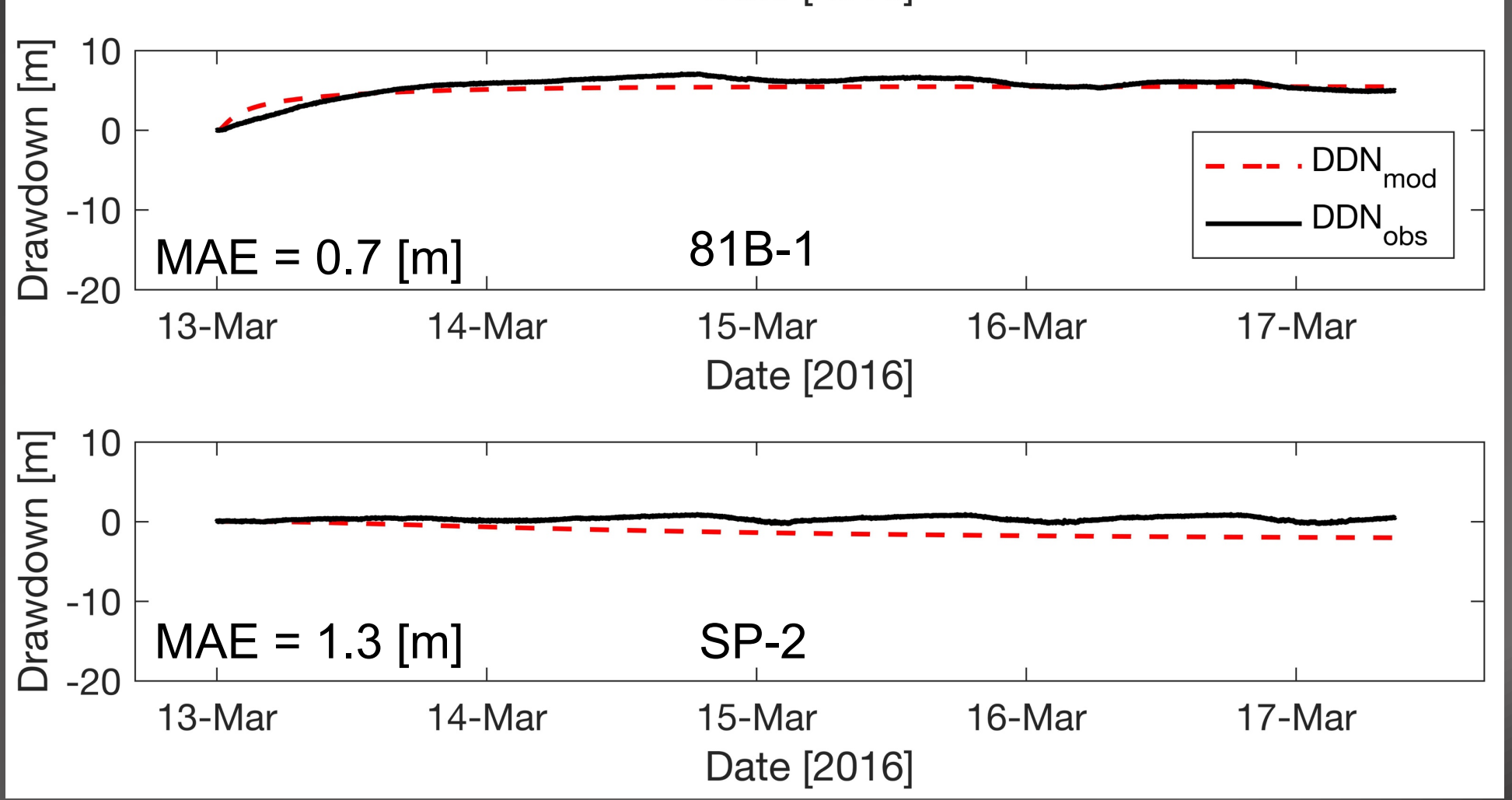




\section{HYDRAULIC CHARACTERIZATION}

\begin{tabular}{|c|c|c|}
\hline Lithology & $\mathrm{K}[\mathrm{m} / \mathrm{s}]$ & $\mathrm{S}_{\mathrm{s}}\left[\mathrm{m}^{-1}\right]$ \\
\hline Sedimentary & $4.1 \times 10^{-2}$ & $7.0 \times 10^{-8}$ \\
\hline Volcanic & $2.7 \times 10^{-4}$ & $3.5 \times 10^{-8}$ \\
\hline Fault & $1.7 \times 10^{-8}$ & $1.8 \times 10^{-6}$ \\
\hline
\end{tabular}

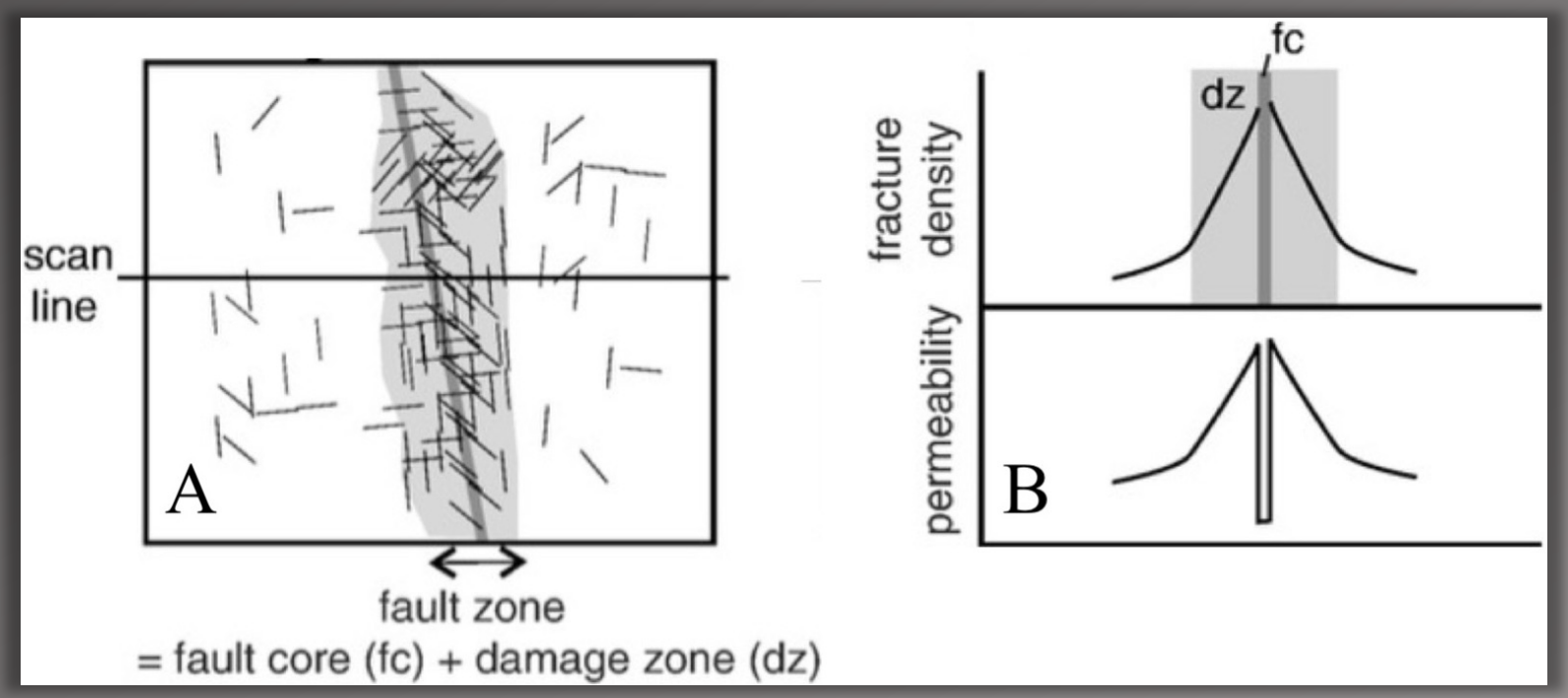

Two component fault zone

- Low permeability fault core

- Higher permeability damage zone

Adapted from Bense et al., [2013] 


\section{FLOW CHARACTERIZATION}

Plan View (100 m depth)

- Advective transport from injection well

- Flow paths show planar flow following fault traces

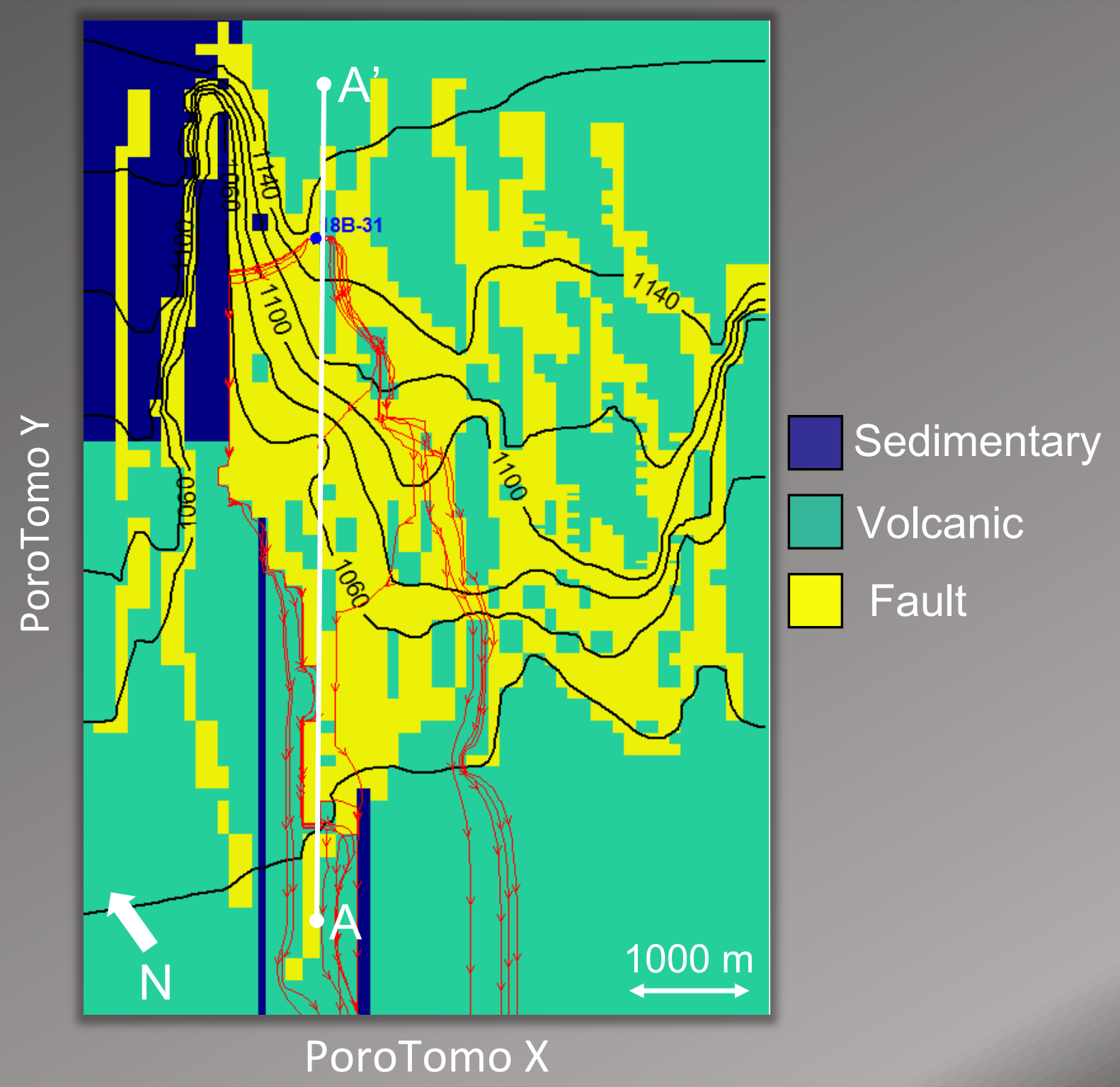




\section{FLOW CHARACTERIZATION}

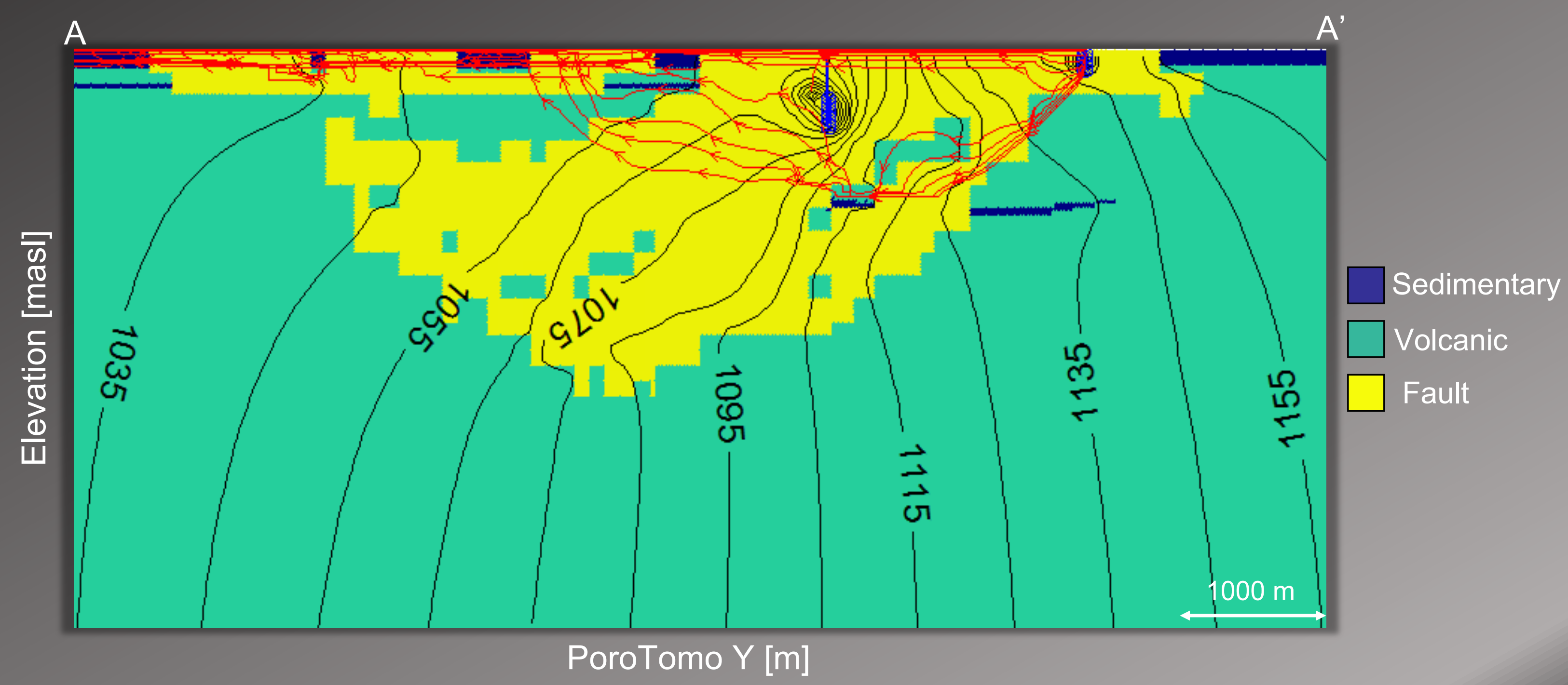




\section{CONCLUSIONS}

- Thermal characterization determines thermal diffusivity estimates which are consistent with expected values based on rock types.

- Hydraulic characterization implies individual faults act as barriers to groundwater flow

- Geophysical, visual observations, and advective transport simulations indicate fault-driven groundwater flow system. 


\section{FUTURE WORK}

Analytical Modeling

- Energy consumed vs energy produced?

- Hydraulically active faults?

- Parameter sensitivity?
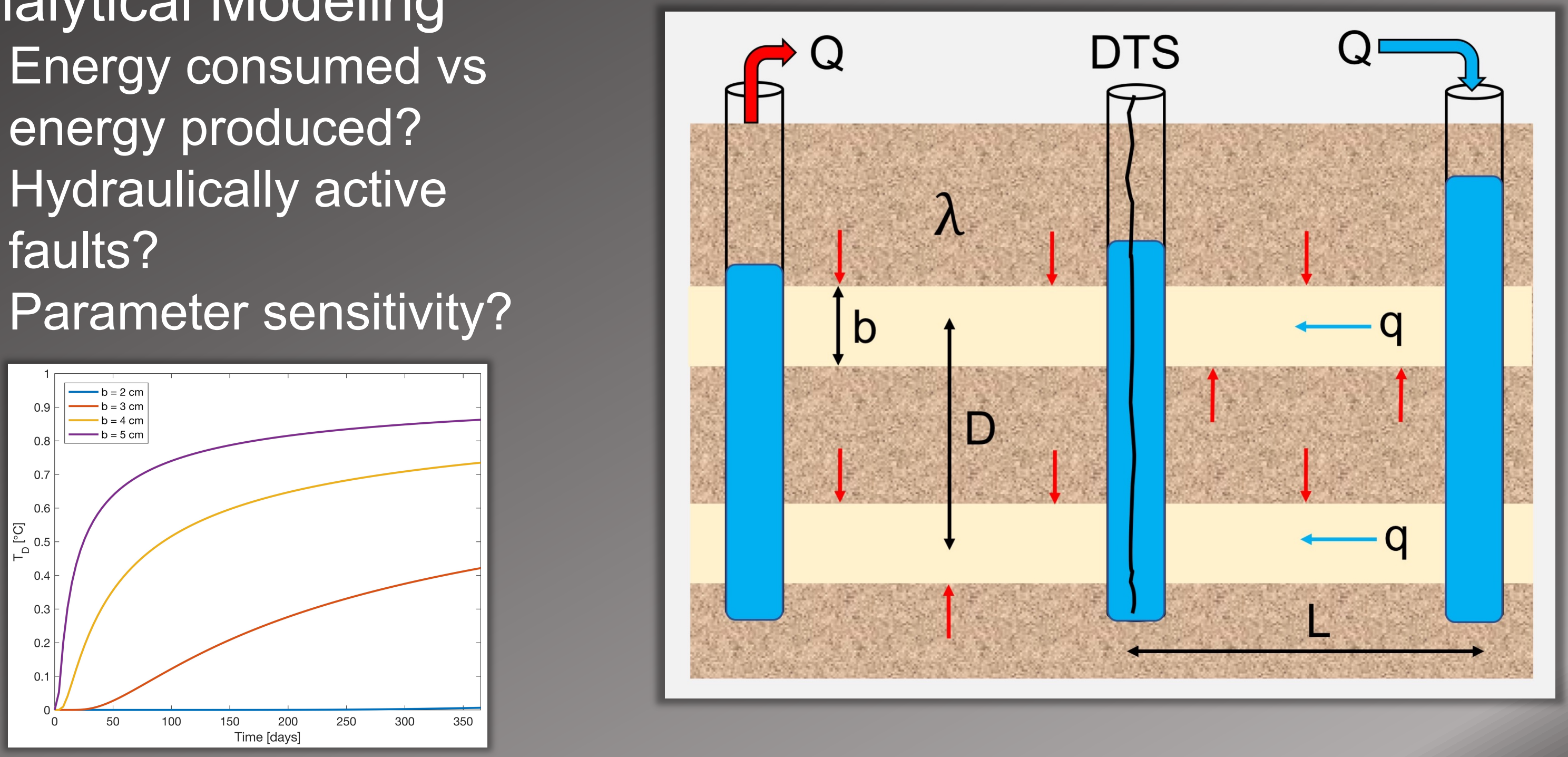


\section{Questions?}

